\title{
Author Index Volume 22 (2010)
}

The issue number is given in front of the pagination

Abe, K., see Yang, J. (1) 225-234

Ábrahám, C.S., see Deli, M.A. (3) 777-794

Adam, J.-F., see Ancelin, M.-L. (S3) S105-S113

Adversi, M., see Di Nino, G. (S3) S121-S127

Agosti, C., see Borroni, B. (1) 195-203

Agosti, C., see Padovani, A. (3) 923-931

Ahuja, M., see Mandal, P.K. (S3) S27-S34

Aisa, B., see Gil-Bea, F.J. (3) 829-838

Ala, T., see Struble, R.G. (2) 393-399

Alberici, A., see Borroni, B. (1) 195-203

Albertini, V., see Ghidoni, R. (3) 985-991

Alcolea, D., see Samaranch, L. (4) 1065-1071

Aldudo, J., see Martínez-García, A. (4) 1181-1187

Alldred, M.J., see Ginsberg, S.D. (2) 631-639

Almeida, O.F.X., see Liu, J. (2) 541-556

Alonso, A., see Samaranch, L. (4) 1065-1071

Alpérovitch, A., see Lamberta, J.-C. (1) 247-255

Álvarez, S., see Blanco, A. (2) 493-505

Alvarez, V., see Lamberta, J.-C. (1) 247-255

Amouyel, P., see Lamberta, J.-C. (1) 247-255

An, Y., see Thambisetty, M. (4) 1099-1109

Ancín, I., see Samaranch, L. (4) 1065-1071

Ancelin, M.-L., G. de Roquefeuil, J. Scali, F. Bonnel, J.-F. Adam, J.-C. Cheminal, J.-P. Cristol, A.M. Dupuy, I. Carrière and K. Ritchie, Long-Term Post-Operative Cognitive Decline in the Elderly: The Effects of Anesthesia Type, Apolipoprotein E Genotype, and Clinical Antecedents (S3) S105S113

Andreasson, U., see Chiasserini, D. (4) 1281-1288

Annoni, G., see Lamberta, J.-C. (1) 247-255

Antunez, C., see Lamberta, J.-C. (1) 247-255

Arai, K., see Yoshiyama, Y. (1) 295-306

Arbel-Ornath, M., M. Becker, P. Rabinovich-Toidman, M. Gartner and B. Solomon, Immunomodulation of $\mathrm{A} \beta \mathrm{PP}$ Processing Alleviates Amyloid- $\beta$ Related Pathology in Alzheimer's Disease Transgenic Mice (2) 469-482

Archetti, S., see Padovani, A. (3) 923-931
Arenaza-Urquijo, E.M., see Sala-Llonch, R. (2) 523539

Arnim, C.A.F.v., see Steinacker, P. (1) 119-128

Arosio, B., see Lamberta, J.-C. (1) 247-255

Arrang, J.-M., see Motawaj, M. (3) 861-871

Astarita, G., see Murphy, M.P. (1) 135-150

Avila, J., see Gahete, M.D. (3) 819-828

Avni, S., see Israeli-Korn, S.D. (3) 1005-1013

Ayala, P., see Subramanian, S. (2) 619-629

Babiloni, C., R. Lizio, F. Vecchio, G.B. Frisoni, M. Pievani, C. Geroldi, F. Claudia, R. Ferri, B. Lanuzza and P.M. Rossini, Reactivity of Cortical Alpha Rhythms to Eye Opening in Mild Cognitive Impairment and Alzheimer's Disease: an EEG Study (4) 1047-1064

Bahar-Fuchs, A., G. Chételat, V.L. Villemagne, S. Moss, K. Pike, C.L. Masters, C. Rowe and G. Savage, Olfactory Deficits and Amyloid- $\beta$ Burden in Alzheimer's Disease, Mild Cognitive Impairment, and Healthy Aging: A PiB PET Study (4) 10811087

Bajo, R., F. Maestú, A. Nevado, M. Sancho, R. Gutiérrez, P. Campo, N.P. Castellanos, P. Gil, S. Moratti, E. Pereda and F. del-Pozo, Functional Connectivity in Mild Cognitive Impairment During a Memory Task: Implications for the Disconnection Hypothesis (1) 183-193

Baker, L.D., L.L. Frank, K. Foster-Schubert, P.S. Green, C.W. Wilkinson, A. McTiernan, B.A. Cholerton, S.R. Plymate, M.A. Fishel, G.S. Watson, G.E. Duncan, P.D. Mehta and S. Craft, Aerobic Exercise Improves Cognition for Older Adults with Glucose Intolerance, A Risk Factor for Alzheimer's Disease (2) 569-579

Baltrons, M.A., see Lichtenstein, M.P. (4) 1135-1155

Bandy, D., see Tzimopoulou, S. (4) 1241-1256

Barabash, A., see Samaranch, L. (4) 1065-1071

Barbeau, E., see Didic, M. (4) 1269-1279 
Barbieri, P., see Nobili, F. (3) 993-1003

Barcikowska, M., see Gabryelewicz, T. (4) 1123-1133

Barczak, A., see Gabryelewicz, T. (4) 1123-1133

Bargalló, N., see Fortea, J. (3) 909-922

Bargalló, N., see Sala-Llonch, R. (2) 523-539

Barini, E., see Borghi, R. (3) 771-775

Barrett, E., see Murphy, M.P. (1) 135-150

Bartenstein, P., see Förster, S. (2) 581-591

Bartrés-Faz, D., see Fortea, J. (3) 909-922

Bartrés-Faz, D., see Sala-Llonch, R. (2) 523-539

Bartsch, A., see Haller, S. (1) 315-327

Basun, H., see Sundelöf, J. (4) 1223-1230

Beach, T.G., see Valla, J. (1) 307-313

Becker, M., see Arbel-Ornath, M. (2) 469-482

Becker, R.E., see Winblad, B. (4) 1201-1208

Beckett, T.L., see Murphy, M.P. (1) 135-150

Bednar, M.M., see Vanderweyde, T. (S3) S91-S104

Beecham, G.W., see Lamberta, J.-C. (1) 247-255

Belden, C.M., see Sabbagh, M.N. (3) 1015-1021

Belinson, H., Z. Kariv-Inbal, R. Kayed, E. Masliah and D.M. Michaelson, Following Activation of the Amyloid Cascade, Apolipoprotein E4 Drives the in vivo Oligomerization of Amyloid- $\beta$ Resulting in Neurodegeneration (3) 959-970

Bellelli, G., see Padovani, A. (3) 923-931

Belmar, S., see Ill-Raga, G. (2) 641-652

Benito-León, J., A.J. Mitchell, S. Vega and F. BermejoPareja, A Population-Based Study of Cognitive Function in Older People with Subjective Memory Complaints (1) 159-170

Benito-León, J., see Bermejo-Pareja, F. (3) 949-958

Benito-León, J., see Cacho, J. (3) 889-896

Bennet, A.M., C.A. Reynolds, M. Gatz, K. Blennow, N.L. Pedersen and J.A. Prince, Pleiotropy in the Presence of Allelic Heterogeneity: Alternative Genetic Models for the Influence of $A P O E$ on Serum LDL, CSF Amyloid- $\beta_{42}$, and Dementia (1) $129-134$

Benussi, L., see Ghidoni, R. (3) 985-991

Berdynski, M., see Gabryelewicz, T. (4) 1123-1133

Berendse, H.W., see Reesink, F.E. (1) 87-95

Berg, D., see Maetzler, W. (3) 933-938

Bermejo-Pareja, F., J. Benito-León, E.D. Louis, R. Trincado, E. Carro, A. Villarejo and A.G. de la Cámara, Risk of Incident Dementia in DrugUntreated Arterial Hypertension: A PopulationBased Study (3) 949-958

Bermejo-Pareja, F., see Benito-León, J. (1) 159-170

Bermejo-Pareja, F., see Martínez-García, A. (4) 11811187

Berr, C., see Lamberta, J.-C. (1) 247-255
Bertram, L., see Lamberta, J.-C. (1) 247-255

Bettens, K., see Lamberta, J.-C. (1) 247-255

Beyer, N., see Coulson, D.T.R. (4) 1111-1122

Bian, J.-S., see Liu, Y.-Y. (4) 1189-1200

Bigni, B., see Padovani, A. (3) 923-931

Bilbao, J.M., see Gabryelewicz, T. (4) 1123-1133

Bilotta, F., A. Doronzio, E. Stazi, L. Titi, V. Fodale, G. Di Nino and G. Rosa, Postoperative Cognitive Dysfunction: Toward the Alzheimer's Disease Pathomechanism Hypothesis (S3) S81-S89

Binetti, G., see Ghidoni, R. (3) 985-991

Bird, N.P., see Tzimopoulou, S. (4) 1241-1256

Black, S.E., see Gabryelewicz, T. (4) 1123-1133

Blake, J., see Tierney, M.C. (4) 1331-1338

Blanco, A., S. Álvarez, M. Fresno and M.Á. MuñozFernández, Amyloid- $\beta$ Induces Cyclooxygenase2 and $\mathrm{PGE}_{2}$ Release in Human Astrocytes in NF- $\kappa$ B Dependent Manner (2) 493-505

Blankenstein, M.A., see Mulder, S.D. (4) 1073-1079

Blankenstein, M.A., see Reesink, F.E. (1) 87-95

Blennow, K., see Bennet, A.M. (1) 129-134

Blennow, K., see Chiasserini, D. (4) 1281-1288

Blennow, K., see Sundelöf, J. (4) 1223-1230

Blesa, R., see Samaranch, L. (4) 1065-1071

Blobner, M., see Eckel, B. (S3) S21-S26

Boada, M., see Lamberta, J.-C. (1) 247-255

Bonetti, M., see Prestia, A. (4) 1339-1349

Bonnel, F., see Ancelin, M.-L. (S3) S105-S113

Bonuccelli, U., see Borroni, B. (1) 195-203

Borghi, R., A. Piccini, E. Barini, G. Cirmena, M. Guglielmotto, E. Tamagno, M. Fornaro, G. Perry, M.A. Smith, A. Garuti and M. Tabaton, Upregulation of Presenilin 1 in Brains of Sporadic, Late-Onset Alzheimer's Disease (3) 771-775

Borro, M., R.A. Cavallaro, G. Gentile, V. Nicolia, A. Fuso, M. Simmaco and S. Scarpa, One-Carbon Metabolism Alteration Affects Brain Proteome Profile in a Mouse Model of Alzheimer's Disease (4) $1257-1268$

Borroni, B., M. Malinverno, F. Gardoni, M. Grassi, L. Parnetti, C. Agosti, A. Alberici, E. Premi, U. Bonuccelli, R. Gasparotti, P. Calabresi, M. Di Luca and A. Padovani, Combining CSF Tau form Ratio and MRI Midbrain to Pons Measure to Identify Early Progressive Supranuclear Palsy (1) 195-203

Borroni, B., see Padovani, A. (3) 923-931

Bosch, B., see Fortea, J. (3) 909-922

Bosch, B., see Sala-Llonch, R. (2) 523-539

Bosch-Morató, M., see Ill-Raga, G. (2) 641-652

Bosco, P., see Lamberta, J.-C. (1) 247-255

Bossù, P., see Lamberta, J.-C. (1) 247-255 
Boucau, M.-C., see Candela, P. (3) 849-859

Boulanger, E., see Candela, P. (3) 849-859

Bredesen, D.E., V. John and V. Galvan, Importance of the Caspase Cleavage Site in Amyloid- $\beta$ Protein Precursor (1) 57-63

Brewer, G.J., see Parihar, M.S. (3) 741-763

Brewer, G.J., see Struble, R.G. (2) 393-399

Brice, A., see Broustal, O. (3) 765-769

Brockbank, S., see Coulson, D.T.R. (4) 1111-1122

Brockmann, K., see Maetzler, W. (3) 933-938

Broustal, O., A. Camuzat, L. Guillot-Noël, N. Guy, S. Millecamps, D. Deffond, L. Lacomblez, V. Golfier, D. Hannequin, F. Salachas, W. Camu, M. Didic, B. Dubois, V. Meininger, I. Le Ber, A. Brice and the French clinical and genetic research network on FTD/FTD-MND, FUS Mutations in Frontotemporal Lobar Degeneration with Amyotrophic Lateral Sclerosis (3) 765-769

Brouwers, N., see Lamberta, J.-C. (1) 247-255

Brown, A.P., see Tzimopoulou, S. (4) 1241-1256

Browne, A., see Zhang, C. (2) 683-694

Bruce-Keller, A.J., see Ebenezer, P.J. (3) 839-848

Brugnolo, A., see Nobili, F. (3) 993-1003

Bruinsma, G., see Winblad, B. (4) 1201-1208

Buerger, K., see Förster, S. (2) 581-591

Bullido, M.J., see Lamberta, J.-C. (1) 247-255

Bullido, M.J., see Martínez-García, A. (4) 1181-1187

Burr, O., see Rosengarten, B. (2) 415-421

Cabranes, J.A., see Samaranch, L. (4) 1065-1071

Cacho, J., J. Benito-León, R. García-García, B. Fernández-Calvo, J.L. Vicente-Villardón and A.J. Mitchell, Does the Combination of the MMSE and Clock Drawing Test (Mini-Clock) Improve the Detection of Mild Alzheimer's Disease and Mild Cognitive Impairment? (3) 889-896

Calabresi, P., see Borroni, B. (1) 195-203

Calabresi, P., see Chiasserini, D. (4) 1281-1288

Callebert, J., see Motawaj, M. (3) 861-871

Campbell, J., see Thambisetty, M. (4) 1099-1109

Campion, D., see Lamberta, J.-C. (1) 247-255

Campo, P., see Bajo, R. (1) 183-193

Camu, W., see Broustal, O. (3) 765-769

Camuzat, A., see Broustal, O. (3) 765-769

Candela, P., F. Gosselet, J. Saint-Pol, E. Sevin, M.-C. Boucau, E. Boulanger, R. Cecchelli and L. Fenart, Apical-to-Basolateral Transport of Amyloid$\beta$ Peptides through Blood-Brain Barrier Cells is Mediated by the Receptor for Advanced Glycation End-Products and is Restricted by P-Glycoprotein (3) 849-859
Cao, Y., Y. Xiao, R. Ravid and Z.-Z. Guan, Changed Clathrin Regulatory Proteins in the Brains of Alzheimer's Disease Patients and Animal Models (1) 329-342

Carrière, I., see Ancelin, M.-L. (S3) S105-S113

Carriba, P., see Lichtenstein, M.P. (4) 1135-1155

Carro, E., see Bermejo-Pareja, F. (3) 949-958

Carro, E., see Martínez-García, A. (4) 1181-1187

Caselli, R.J., see Valla, J. (1) 307-313

Castaño, J.P., see Gahete, M.D. (3) 819-828

Castellanos, N.P., see Bajo, R. (1) 183-193

Castiglia, M., see Tzimopoulou, S. (4) 1241-1256

Cavallaro, R.A., see Borro, M. (4) 1257-1268

Ceccaldi, M., see Didic, M. (4) 1269-1279

Cecchelli, R., see Candela, P. (3) 849-859

Cedazo-Mínguez, A., see Gil-Bea, F.J. (2) 405-413

Cedazo-Mínguez, A., see Gil-Bea, F.J. (3) 829-838

Cerini, C., see Padovani, A. (3) 923-931

Cerpa, W., see Ill-Raga, G. (2) 641-652

Cervantes, S., see Samaranch, L. (4) 1065-1071

Chabriat, H., see Zhu, Y.-C. (2) 663-672

Chang, L., see Liu, J. (2) 541-556

Chang, Y.-H., see Na, H.-R. (1) 151-158

Chauhan, A., see Ji, L. (2) 609-617

Chauhan, V., see Ji, L. (2) 609-617

Chauviré, V., see Sarazin, M. (1) 285-294

Che, S., see Ginsberg, S.D. (2) 631-639

Cheminal, J.-C., see Ancelin, M.-L. (S3) S105-S113

Chen, K., see Tzimopoulou, S. (4) 1241-1256

Chen, S., K. Townsend, T.E. Goldberg, P. Davies and C. Conejero-Goldberg, MAPT Isoforms: Differential Transcriptional Profiles Related to 3R and 4R Splice Variants (4) 1313-1329

Chen, X., J.F. Wagener, D.H. Morgan, L. Hui, O. Ghribi and J.D. Geiger, Endolysosome Mechanisms Associated with Alzheimer's Disease-like Pathology in Rabbits Ingesting Cholesterol-Enriched Diet (4) 1289-1303

Chételat, G., see Bahar-Fuchs, A. (4) 1081-1087

Chiasserini, D., L. Parnetti, U. Andreasson, H. Zetterberg, D. Giannandrea, P. Calabresi and K. Blennow, CSF Levels of Heart Fatty Acid Binding Protein are Altered During Early Phases of Alzheimer's Disease (4) 1281-1288

Cho, S.-T., see Na, H.-R. (1) 151-158

Cholerton, B.A., see Baker, L.D. (2) 569-579

Chupin, M., see Sarazin, M. (1) 285-294

Cirmena, G., see Borghi, R. (3) 771-775

Clarimón, J., see Samaranch, L. (4) 1065-1071

Claudia, F., see Babiloni, C. (4) 1047-1064 
Collette, F., M. Van der Linden and E. Salmon, Dissociation between Controlled and Automatic Processes in the Behavioral Variant of Fronto-Temporal Dementia (3) 897-907

Colliot, O., see Sarazin, M. (1) 285-294

Combarros, O., see Lamberta, J.-C. (1) 247-255

Combarros, O., see Martínez-García, A. (4) 1181-1187

Conejero-Goldberg, C., see Chen, S. (4) 1313-1329

Confort-Gouny, S., see Didic, M. (4) 1269-1279

Connor, D.J., see Sabbagh, M.N. (3) 1015-1021

Cooper, A., see Westman, E. (1) 171-181

Córdoba-Chacón, J., see Gahete, M.D. (3) 819-828

Corneveaux, J.J., see Valla, J. (1) 307-313

Cosseddu, M., see Padovani, A. (3) 923-931

Coto, E., see Lamberta, J.-C. (1) 247-255

Coulson, D.T.R., N. Beyer, J.G. Quinn, S. Brockbank, J. Hellemans, G.B. Irvine, R. Ravid and J.A. Johnston, BACE1 mRNA Expression in Alzheimer's Disease Postmortem Brain Tissue (4) 1111-1122

Counts, S.E., see Ginsberg, S.D. (2) 631-639

Cozzone, P., see Didic, M. (4) 1269-1279

Craft, S., see Baker, L.D. (2) 569-579

Cristol, J.-P., see Ancelin, M.-L. (S3) S105-S113

Csete, M., see Deli, M.A. (3) 777-794

Csiszár, B., see Deli, M.A. (3) 777-794

Cumming, P., see Förster, S. (2) 581-591

Cunningham, V.J., see Tzimopoulou, S. (4) 1241-1256

Curth, A., see Mutter, J. (2) 357-374

Cutler, R.G., see Liu, D. (2) 443-457

Czyzewski, K., see Gabryelewicz, T. (4) 1123-1133

Dangour, A.D., P.J. Whitehouse, K. Rafferty, S.A. Mitchell, L. Smith, S. Hawkesworth and B. Vellas, B-Vitamins and Fatty Acids in the Prevention and Treatment of Alzheimer's Disease and Dementia: A Systematic Review (1) 205-224

Dannhardt, V., see Rosengarten, B. (2) 415-421

Dartigues, J.-F., see Lamberta, J.-C. (1) 247-255

Dasuri, K., see Ebenezer, P.J. (3) 839-848

Davies, P., see Chen, S. (4) 1313-1329

Davis, K., see Sabbagh, M.N. (3) 1015-1021

De Deyn, P., see Lamberta, J.-C. (1) 247-255

de Gracia Blanco, M., see Garre-Olmo, J. (4) 11571167

de Gracia Blanco, M., see Garre-Olmo, J. (4) 11691180

de la Cámara, A.G., see Bermejo-Pareja, F. (3) 949-958

de Roquefeuil, G., see Ancelin, M.-L. (S3) S105-S113

de Souza, L.C., see Sarazin, M. (1) 285-294

de Waal, R.M.W., see Timmer, N.M. (2) 345-355

de Yébenes, J.G., see Mena, M.Á. (S3) S43-S48
Deffond, D., see Broustal, O. (3) 765-769

Degerman-Gunnarsson, M., see Sundelöf, J. (4) 12231230

Dekel, B.G.S., see Di Nino, G. (S3) S121-S127

DeKosky, S.T., see Lamberta, J.-C. (1) 247-255

del Carmen Mugueta, M., see Gil-Bea, F.J. (3) 829-838

Del Zompo, M., see Lamberta, J.-C. (1) 247-255

Delepine, M., see Lamberta, J.-C. (1) 247-255

Deli, M.A., S. Veszelka, B. Csiszár, A. Tóth, Á. Kittel, M. Csete, Á. Sipos, A. Szalai, L. Fülöp, B. Penke, C.S. Ábrahám and M. Niwa, Protection of the Blood-Brain Barrier by Pentosan Against Amyloid- $\beta$-Induced Toxicity (3) 777-794

del-Pozo, F., see Bajo, R. (1) 183-193

Desenzani, S., see Ghidoni, R. (3) 985-991

Dessi, B., see Nobili, F. (3) 993-1003

Deth, R., see Mutter, J. (2) 357-374

Di Luca, M., see Borroni, B. (1) 195-203

Di Nino, G., M. Adversi, B.G.S. Dekel, V. Fodale, G. Rosa and R.M. Melotti, Peri-Operative Risk Management in Patients with Alzheimer's Disease (S3) S121-S127

Di Nino, G., see Bilotta, F. (S3) S81-S89

Di Nino, G., see Schifilliti, D. (S3) S35-S41

Didic, M., J.-P. Ranjeva, E. Barbeau, S. Confort-Gouny, Y. Le Fur, O. Felician, J. Mancini, M. Poncet, M. Ceccaldi and P. Cozzone, Impaired Visual Recognition Memory in Amnestic Mild Cognitive Impairment is Associated with Mesiotemporal Metabolic Changes on Magnetic Resonance Spectroscopic Imaging (4) 1269-1279

Didic, M., see Broustal, O. (3) 765-769

Ding, D., see Zhou, B. (2) 673-682

Ding, K., see Lu, Q. (1) 235-245

Diniz, B.S., A.L. Teixeira, E.B. Ojopi, L.L. Talib, V.A. Mendonça, W.F. Gattaz and O.V. Forlenza, Higher Serum sTNFR1 Level Predicts Conversion from Mild Cognitive Impairment to Alzheimer's Disease (4) 1305-1311

DiVito, J.R., see Zhang, C. (2) 683-694

Dixon, I.J., see Tzimopoulou, S. (4) 1241-1256

Dong, Y., see Zhang, C. (2) 683-694

Doronzio, A., see Bilotta, F. (S3) S81-S89

Double, K.L., see Schofield, E.C. (3) 981-984

Dowling, A.L.S., see Murphy, M.P. (1) 135-150

Drago, V., see Prestia, A. (4) 1339-1349

Du, X.-Y., see Yang, S.-G. (1) 107-117

Dubois, B., see Broustal, O. (3) 765-769

Dubois, B., see Sarazin, M. (1) 285-294

Dufouil, C., see Zhu, Y.-C. (2) 663-672

Duncan, G.E., see Baker, L.D. (2) 569-579 
Dupuy, A.-M., see Ancelin, M.-L. (S3) S105-S113

Ebenezer, P.J., A.M. Weidner, H. LeVine, III, W.R. Markesbery, M.P. Murphy, L. Zhang, K. Dasuri, S.O. Fernandez-Kim, A.J. Bruce-Keller, E. Gavilán and J.N. Keller, Neuron Specific Toxicity of Oligomeric Amyloid- $\beta$ : Role for JUN-Kinase and Oxidative Stress (3) 839-848

Eckel, B., M. Blobner and G. Rammes, Anesthetics Promoting in vitro $\mathrm{A} \beta \mathrm{PP}$ Metabolism and Amyloid- $\beta$ Toxicity (S3) S21-S26

Eikelenboom, P., see Richard, E. (3) 811-818

Eling, P., see Haldenwanger, A. (3) 971-980

Ellis, G., E. Fang, M. Maheshwari, E. Roltsch, L. Holcomb, D. Zimmer, D. Martinez and I.V.J. Murray, Lipid Oxidation and Modification of Amyloid- $\beta$ (A $\beta$ ) in vitro and in vivo (2) 593-607

Emanuele, E., see Ghidoni, R. (3) 985-991

Emch, J., see Haller, S. (1) 315-327

Engelborghs, S., see Lamberta, J.-C. (1) 247-255

Epelbaum, J., see Lamberta, J.-C. (1) 247-255

Erb, M., see Saur, R. (1) 267-284

Eriksdotter-Jönhagen, M., see Sundelöf, J. (4) 12231230

Eschweiler, G.W., see Saur, R. (1) 267-284

Fagan, T., see Landhuis, E. (3) 1033-1037

Fang, E., see Ellis, G. (2) 593-607

Farrer, L.A., see Israeli-Korn, S.D. (3) 1005-1013

Felician, O., see Didic, M. (4) 1269-1279

Felipo, V., see Monfort, P. (3) 795-809

Fenart, L., see Candela, P. (3) 849-859

Feng, Y., see Yang, S.-G. (1) 107-117

Fernández-Calvo, B., see Cacho, J. (3) 889-896

Fernandez-Kim, S.O., see Ebenezer, P.J. (3) 839-848

Ferri, R., see Babiloni, C. (4) 1047-1064

Ferri, R., see Lamberta, J.-C. (1) 247-255

Ferrucci, L., see Thambisetty, M. (4) 1099-1109

Fiévet, N., see Lamberta, J.-C. (1) 247-255

Fishel, M.A., see Baker, L.D. (2) 569-579

Foa, L., see Vincent, A.J. (3) 699-714

Fodale, V., K. Ritchie, L.S. Rasmussen and P.K. Mandal, Preface: Anesthetics and Alzheimer's Disease: Background and Research (S3) S1-S3

Fodale, V., see Bilotta, F. (S3) S81-S89

Fodale, V., see Di Nino, G. (S3) S121-S127

Fodale, V., see Mandal, P.K. (S3) S135-S136

Fodale, V., see Schifilliti, D. (S3) S35-S41

Forlenza, O.V., see Diniz, B.S. (4) 1305-1311

Forman, S.A., see Vanderweyde, T. (S3) S91-S104

Fornaro, M., see Borghi, R. (3) 771-775
Förster, S., A. Vaitl, S.J. Teipel, I. Yakushev, M. Mustafa, C. la Fougère, A. Rominger, P. Cumming, P. Bartenstein, H. Hampel, T. Hummel, K. Buerger, W. Hundt and S. Steinbach, Functional Representation of Olfactory Impairment in Early Alzheimer's Disease (2) 581-591

Fortea, J., R. Sala-Llonch, D. Bartrés-Faz, B. Bosch, A. Lladó, N. Bargalló, J.L. Molinuevo and R. Sánchez-Valle, Increased Cortical Thickness and Caudate Volume Precede Atrophy in PSEN Mutation Carriers (3) 909-922

Forti, P., see Lamberta, J.-C. (1) 247-255

Foster-Schubert, K., see Baker, L.D. (2) 569-579

Foy, C., see Westman, E. (1) 171-181

Frank, A., see Martínez-García, A. (4) 1181-1187

Frank, L.L., see Baker, L.D. (2) 569-579

Frank-Garcia, A., see Lamberta, J.-C. (1) 247-255

Fraser, T., see Tayler, H. (4) 1363-1373

Fresno, M., see Blanco, A. (2) 493-505

Friedhoff, L.T., see Winblad, B. (4) 1201-1208

Friedland, R.P., see Israeli-Korn, S.D. (3) 1005-1013

Frisardi, V., F. Panza, D. Seripa, B.P. Imbimbo, G. Vendemiale, A. Pilotto and V. Solfrizzi, Nutraceutical Properties of Mediterranean Diet and Cognitive Decline: Possible Underlying Mechanisms (3) 715-740

Frisoni, G.B., see Babiloni, C. (4) 1047-1064

Frisoni, G.B., see Prestia, A. (4) 1339-1349

Frölich, L., see Winblad, B. (4) 1201-1208

Frommann, I., see Popp, J. (2) 459-468

Frosch, M.P., see Lu, Q. (1) 235-245

Frost, S., R.N. Martins and Y. Kanagasingam, Biomarkers for Early Detection of Alzheimer's Disease (1) $1-16$

Fukushima, M., see Zhou, B. (2) 673-682

Fülöp, L., see Deli, M.A. (3) 777-794

Funder, K.S., J. Steinmetz and L.S. Rasmussen, Anesthesia for the Patient with Dementia (S3) S129S134

Furukawa, K., see Liu, D. (2) 443-457

Fuso, A., see Borro, M. (4) 1257-1268

Fuso, A., see Milagre, I. (4) 1209-1221

Gabryelewicz, T., M. Masellis, M. Berdynski, J.M. Bilbao, E. Rogaeva, P. St. George-Hyslop, A. Barczak, K. Czyzewski, M. Barcikowska, Z. Wszolek, S.E. Black and C. Zekanowski, IntraFamilial Clinical Heterogeneity due to FTLD-U with TDP-43 Proteinopathy Caused by a Novel Deletion in Progranulin Gene (PGRN) (4) 11231133 
Gahete, M.D., A. Rubio, J. Córdoba-Chacón, F. GraciaNavarro, R.D. Kineman, J. Avila, R.M. Luque and J.P. Castaño, Expression of the Ghrelin and Neurotensin Systems is Altered in the Temporal Lobe of Alzheimer's Disease Patients (3) 819-828

Galea, E., see Lichtenstein, M.P. (4) 1135-1155

Galimberti, D., see Lamberta, J.-C. (1) 247-255

Galvan, V., see Bredesen, D.E. (1) 57-63

Gama, M.J., see Milagre, I. (4) 1209-1221

Gao, X., see Liu, J. (2) 541-556

García, A., see Lichtenstein, M.P. (4) 1135-1155

García-García, R., see Cacho, J. (3) 889-896

Gardoni, F., see Borroni, B. (1) 195-203

Garnero, L., see Sarazin, M. (1) 285-294

Garre-Olmo, J., S. López-Pousa, J. Vilalta-Franch, M. de Gracia Blanco and A.B. Vilarrasa, Grouping and Trajectories of Neuropsychiatric Symptoms in Patients with Alzheimer's Disease. Part II: TwoYear Patient Trajectories (4) 1169-1180

Garre-Olmo, J., S. López-Pousa, J. Vilalta-Franch, M. de Gracia Blanco and A.B. Vilarrasa, Grouping and Trajectories of the Neuropsychiatric Symptoms in Patients with Alzheimer's Disease, Part I: Symptom Clusters (4) 1157-1167

Gartner, M., see Arbel-Ornath, M. (2) 469-482

Garuti, A., see Borghi, R. (3) 771-775

Gasparotti, R., see Borroni, B. (1) 195-203

Gasperini, R., see Vincent, A.J. (3) 699-714

Gasser, T., see Maetzler, W. (3) 933-938

Gattaz, W.F., see Diniz, B.S. (4) 1305-1311

Gatz, M., see Bennet, A.M. (1) 129-134

Gavilán, E., see Ebenezer, P.J. (3) 839-848

Geiger, J.D., see Chen, X. (4) 1289-1303

Gentile, G., see Borro, M. (4) 1257-1268

George-Hyslop, P.St., see Gabryelewicz, T. (4) 11231133

Gerardin, E., see Sarazin, M. (1) 285-294

Geroldi, C., see Babiloni, C. (4) 1047-1064

Ghidoni, R., L. Benussi, M. Glionna, S. Desenzani, V. Albertini, E. Levy, E. Emanuele and G. Binetti, Plasma Cystatin C and Risk of Developing Alzheimer's Disease in Subjects with Mild Cognitive Impairment (3) 985-991

Ghribi, O., see Chen, X. (4) 1289-1303

Giacobini, E., see Winblad, B. (4) 1201-1208

Giannakopoulos, P., see Haller, S. (1) 315-327

Giannandrea, D., see Chiasserini, D. (4) 1281-1288

Giedraitis, V., see Lamberta, J.-C. (1) 247-255

Giedraitis, V., see Sundelöf, J. (4) 1223-1230

Gil, P., see Bajo, R. (1) 183-193
Gil-Bea, F.J., B. Aisa, A. Solomon, M. Solas, M. del Carmen Mugueta, B. Winblad, M. Kivipelto, A. Cedazo-Mínguez and M.J. Ramírez, HPA Axis Dysregulation Associated to Apolipoprotein E4 Genotype in Alzheimer's Disease (3) 829-838

Gil-Bea, F.J., M. Solas, A. Solomon, C. Mugueta, B. Winblad, M. Kivipelto, M.J. Ramirez and A. Cedazo-Mínguez, Insulin Levels are Decreased in the Cerebrospinal Fluid of Women with Prodomal Alzheimer's Disease (2) 405-413

Ginsberg, S.D., E.J. Mufson, S.E. Counts, J. Wuu, M.J. Alldred, R.A. Nixon and S. Che, Regional Selectivity of rab5 and rab7 Protein Upregulation in Mild Cognitive Impairment and Alzheimer's Disease (2) 631-639

Girtler, N., see Nobili, F. (3) 993-1003

Glionna, M., see Ghidoni, R. (3) 985-991

Godoy, J., see Ill-Raga, G. (2) 641-652

Gold, G., see Haller, S. (1) 315-327

Gold, M., see Tzimopoulou, S. (4) 1241-1256

Goldberg, T.E., see Chen, S. (4) 1313-1329

Golfier, V., see Broustal, O. (3) 765-769

Gómez-Isla, T., see Samaranch, L. (4) 1065-1071

Gong, C.-X., see Run, X. (S3) S49-S55

González-Pérez, A., see Lamberta, J.-C. (1) 247-255

Gosselet, F., see Candela, P. (3) 849-859

Gracia-Navarro, F., see Gahete, M.D. (3) 819-828

Grassi, M., see Borroni, B. (1) 195-203

Green, P.S., see Baker, L.D. (2) 569-579

Greig, N.H., see Liu, D. (2) 443-457

Greig, N.H., see Winblad, B. (4) 1201-1208

Grodd, W., see Saur, R. (1) 267-284

Gu, Y., J.A. Luchsinger, Y. Stern and N. Scarmeas, Mediterranean Diet, Inflammatory and Metabolic Biomarkers, and Risk of Alzheimer's Disease (2) 483-492

Guan, Z.-Z., see Cao, Y. (1) 329-342

Guglielmotto, M., see Borghi, R. (3) 771-775

Guillot-Noël, L., see Broustal, O. (3) 765-769

Guix, F.X., see Ill-Raga, G. (2) 641-652

Guo, Q., see Zhou, B. (2) 673-682

Gutiérrez, R., see Bajo, R. (1) 183-193

Guy, N., see Broustal, O. (3) 765-769

Hack, C.E., see Mulder, S.D. (4) 1073-1079

Haines, J.L., see Lamberta, J.-C. (1) 247-255

Haldenwanger, A., P. Eling, A. Kastrup and H. Hildebrandt, Correlation between Cognitive Impairment and CSF Biomarkers in Amnesic MCI, nonAmnesic MCI, and Alzheimer's Disease (3) 971980 
Haller, S., D. Nguyen, C. Rodriguez, J. Emch, G. Gold, A. Bartsch, K.O. Lovblad and P. Giannakopoulos, Individual Prediction of Cognitive Decline in Mild Cognitive Impairment Using Support Vector Machine-Based Analysis of Diffusion Tensor Imaging Data (1) 315-327

Hallett, W.A., see Tzimopoulou, S. (4) 1241-1256

Halliday, G.M., see Schofield, E.C. (3) 981-984

Hampel, H., see Förster, S. (2) 581-591

Hampel, H., see Teipel, S.J. (2) 507-522

Han, G., see Kim, H.Y. (1) 73-85

Han, I.-W., see Na, H.-R. (1) 151-158

Hannequin, D., see Broustal, O. (3) 765-769

Hannequin, D., see Lamberta, J.-C. (1) 247-255

Hänninen, T., see Paajanen, T. (4) 1089-1097

Hanon, O., see Lamberta, J.-C. (1) 247-255

Hansson, O., see Sundelöf, J. (4) 1223-1230

Harris, C.J., see Subramanian, S. (2) 619-629

Hauser, A.-K., see Maetzler, W. (3) 933-938

Hawkesworth, S., see Dangour, A.D. (1) 205-224

Head, E., see Murphy, M.P. (1) 135-150

Heikkilä, K., see Virta, J.J. (3) 939-948

Helisalmi, S., see Lamberta, J.-C. (1) 247-255

Hellemans, J., see Coulson, D.T.R. (4) 1111-1122

Hildebrandt, H., see Haldenwanger, A. (3) 971-980

Hiltunen, M., see Lamberta, J.-C. (1) 247-255

Ho, L., see Wang, J. (2) 653-661

Hodges, J.R. see Schofield, E.C. (3) 981-984

Holcomb, L., see Ellis, G. (2) 593-607

Hong, H.-S., J.-Y. Hwang, S.-M. Son, Y.-H. Kim, M. Moon and M.-J. Inhee, FK506 Reduces Amyloid Plaque Burden and Induces MMP-9 in A $\beta$ PP/PS1 Double Transgenic Mice (1) 97-105

Hong, Z., see Zhou, B. (2) 673-682

Hooper, N.M., see Whitehouse, I.J. (3) 1023-1031

Hoozemans, J.J.M., see Richard, E. (3) 811-818

Hsu, W.-M., see Kapoor, A. (2) 423-442

Huang, W.-D., see Yu, C.-J. (1) 257-266

Huentelman, M.J., see Valla, J. (1) 307-313

Hugonot-Diener, L., see Sarazin, M. (1) 285-294

Hui, L., see Chen, X. (4) 1289-1303

Hummel, T., see Förster, S. (2) 581-591

Hundt, W., see Förster, S. (2) 581-591

Hwang, J.-Y., see Hong, H.-S. (1) 97-105

Hwang, S.-A., see Na, H.-R. (1) 151-158

Hye, A., see Thambisetty, M. (4) 1099-1109

Ill-Raga, G., E. Ramos-Fernández, F.X. Guix, M. Tajes, M. Bosch-Morató, E. Palomer, J. Godoy, S. Belmar, W. Cerpa, J.W. Simpkins, N.C. Inestrosa and F.J. Muñoz, Amyloid- $\beta$ Peptide Fibrils Induce Nitro-Oxidative Stress in Neuronal Cells (2) 641-652
Imbimbo, B.P., see Frisardi, V. (3) 715-740

Imbimbo, B.P., see Ross, J.S. (2) 401-404

Inestrosa, N.C., see Ill-Raga, G. (2) 641-652

Ingelsson, M., see Lamberta, J.-C. (1) 247-255

Ingelsson, M., see Sundelöf, J. (4) 1223-1230

Inhee, M.-J., see Hong, H.-S. (1) 97-105

Inzelberg, R., see Israeli-Korn, S.D. (3) 1005-1013

Inzhutova, A.I., see Salmina, A.B. (1) 17-36

Irvine, G.B., see Coulson, D.T.R. (4) 1111-1122

Ishikawa, C., see Yoshiyama, Y. (1) 295-306

Israeli-Korn, S.D., M. Massarwa, E. Schechtman, R. Strugatsky, S. Avni, L.A. Farrer, R.P. Friedland and R. Inzelberg, Mild Cognitive Impairment is Associated with Mild Parkinsonian Signs in a Door-to-Door Study (3) 1005-1013

Jackson, C., see Whitehouse, I.J. (3) 1023-1031

Jacobson, S., see Sabbagh, M.N. (3) 1015-1021

Järvenpää, T., see Virta, J.J. (3) 939-948

Jesse, S., see Steinacker, P. (1) 119-128

Jessen, F., see Popp, J. (2) 459-468

Jeter, B., see Tzimopoulou, S. (4) 1241-1256

Ji, L., V. Chauhan, P. Mehta, J. Wegiel, S. Mehta and A. Chauhan, Relationship Between Proteolytically Cleaved Gelsolin and Levels of Amyloid- $\beta$ Protein in the Brains of Down Syndrome Subjects (2) 609-617

Jiang, H., see Liu, D. (2) 443-457

John, V., see Bredesen, D.E. (1) 57-63

Johnston, J.A., see Coulson, D.T.R. (4) 1111-1122

Jones, S., see Lu, Q. (1) 235-245

Junqué, C., see Sala-Llonch, R. (2) 523-539

Jyoti, A., A. Plano, G. Riedel and B. Platt, EEG, Activity, and Sleep Architecture in a Transgenic $\mathrm{A} \beta \mathrm{PP}_{\mathrm{swe}} / \mathrm{PSEN}_{\mathrm{A} 246 \mathrm{E}}$ Alzheimer's Disease Mouse (3) 873-887

Kaji, K., see Tokita, Y. (4) 1351-1361

Kamboh, M.I., see Lamberta, J.-C. (1) 247-255

Kanagasingam, Y., see Frost, S. (1) 1-16

Kapoor, A., W.-M. Hsu, B.-J. Wang, G.-H. Wu, T.-Y. Lin, S.-J. Lee, C.-T. Yen, S.-M. Liang and Y.-F. Liao, Caveolin-1 Regulates $\gamma$-Secretase-Mediated A $\beta$ PP Processing by Modulating Spatial Distribution of $\gamma$-Secretase in Membrane (2) 423-442

Kaprio, J., see Virta, J.J. (3) 939-948

Kariv-Inbal, Z., see Belinson, H. (3) 959-970

Kastrup, A., see Haldenwanger, A. (3) 971-980

Kataria, R., see Sabbagh, M.N. (3) 1015-1021

Kayed, R., see Belinson, H. (3) 959-970

Kehoe, P.G., see Tayler, H. (4) 1363-1373 
Keller, J.N., see Ebenezer, P.J. (3) 839-848

Kilander, L., see Sundelöf, J. (4) 1223-1230

Kim, D.J., see Kim, H.Y. (1) 73-85

Kim, H.Y., Y. Kim, G. Han and D.J. Kim, Regulation of in vitro $\mathrm{A} \beta_{1-40}$ Aggregation Mediated by Small Molecules (1) 73-85

Kim, S.-Y., see Na, H.-R. (1) 151-158

Kim, T.-Y., see Na, H.-R. (1) 151-158

Kim, Y., see Kim, H.Y. (1) 73-85

Kim, Y.-H., see Hong, H.-S. (1) 97-105

Kineman, R.D., see Gahete, M.D. (3) 819-828

Kinkingnéhun, S., see Sarazin, M. (1) 285-294

Kinsey, A., see Thambisetty, M. (4) 1099-1109

Kittel, Á., see Deli, M.A. (3) 777-794

Kivipelto, M., see Gil-Bea, F.J. (2) 405-413

Kivipelto, M., see Gil-Bea, F.J. (3) 829-838

Klafki, H., see Steinacker, P. (1) 119-128

Klein, M., see Reesink, F.E. (1) 87-95

Kohyama, K., see Tokita, Y. (4) 1351-1361

Kojima, A., see Yoshiyama, Y. (1) 295-306

Kölsch, H., see Popp, J. (2) 459-468

Kornhuber, J., see Popp, J. (2) 459-468

Koskenvuo, M., see Virta, J.J. (3) 939-948

Kretzschmar, H.A., see Steinacker, P. (1) 119-128

Kryscio, R.J., see Murphy, M.P. (1) 135-150

Ksiezak-Reding, H., see Wang, J. (2) 653-661

Kuang, C.-X., see Yu, C.-J. (1) 257-266

Kuiperij, H.B., see Timmer, N.M. (2) 345-355

Kusne, Y., see Valla, J. (1) 307-313

Kwok, J., see Schofield, E.C. (3) 981-984

la Fougère, C., see Förster, S. (2) 581-591

Lacomblez, L., see Broustal, O. (3) 765-769

Lahiri, D.K., see Liu, D. (2) 443-457

Lai, R.Y.K., see Tzimopoulou, S. (4) 1241-1256

Lamberta, J.-C., K. Sleegers, A. González-Pérez, M. Ingelsson, G.W. Beecham, M. Hiltunen, O. Combarros, M.J. Bullido, N. Brouwers, K. Bettens, C. Berr, F. Pasquier, F. Richard, S.T. DeKosky, D. Hannequin, J.L. Haines, G. Tognoni, N. Fiévet, J.-F. Dartigues, C. Tzourio, S. Engelborghs, B. Arosio, E. Coto, P. De Deyn, M. Del Zompo, I. Mateo, M. Boada, C. Antunez, J. Lopez-Arrieta, J. Epelbaum, B.-M.M. Schjeide, A. Frank-Garcia, V. Giedraitis, S. Helisalmi, E. Porcellini, A. Pilotto, P. Forti, R. Ferri, M. Delepine, D. Zelenika, M. Lathrop, E. Scarpini, G. Siciliano, V. Solfrizzi, S. Sorbi, G. Spalletta, G. Ravaglia, F. Valdivieso, S. Vepsäläinen, V. Alvarez, P. Bosco, M. Mancuso, F. Panza, B. Nacmias, P. Bossù, O. Hanon, P. Piccardi, G. Annoni, D. Mann, P. Marambaud,
D. Seripa, D. Galimberti, R.E. Tanzi, L. Bertram, C. Lendon, L. Lannfelt, F. Licastro, D. Campion, M.A. Pericak-Vance, H. Soininen, C. Van Broeckhoven, A. Alpérovitch, A. Ruiz, M.I. Kamboh and P. Amouyel, The CALHM1 P86L Polymorphism is a Genetic Modifier of Age at Onset in Alzheimer's Disease: A Meta-Analysis Study (1) 247-255

Lamet, I., see Samaranch, L. (4) 1065-1071

Landhuis, E. and T. Fagan, Meeting Report: Trial News: Woes, New Approaches (3) 1033-1037

Lanford, G.W., see Lu, Q. (1) 235-245

Lannfelt, L., see Lamberta, J.-C. (1) 247-255

Lannfelt, L., see Sundelöf, J. (4) 1223-1230

Lanuzza, B., see Babiloni, C. (4) 1047-1064

Larsson, A., see Sundelöf, J. (4) 1223-1230

Lathrop, M., see Lamberta, J.-C. (1) 247-255

Le Ber, I., see Broustal, O. (3) 765-769

Le Fur, Y., see Didic, M. (4) 1269-1279

Lee, J.-H., see Liu, D. (2) 443-457

Lee, S.-J., see Kapoor, A. (2) 423-442

Lehéricy, S., see Sarazin, M. (1) 285-294

Lehnert, S., see Steinacker, P. (1) 119-128

Lemstra, A.W., see Reesink, F.E. (1) 87-95

Lendon, C., see Lamberta, J.-C. (1) 247-255

LeVine, III, H., see Ebenezer, P.J. (3) 839-848

LeVine, III, H., see Murphy, M.P. (1) 135-150

Levy, E., see Ghidoni, R. (3) 985-991

Lewczuk, P., see Popp, J. (2) 459-468

Leyhe, T., see Saur, R. (1) 267-284

Liang, S.-M., see Kapoor, A. (2) 423-442

Liang, Z., see Run, X. (S3) S49-S55

Liao, Y.-F., see Kapoor, A. (2) 423-442

Licastro, F., see Lamberta, J.-C. (1) 247-255

Lichtenstein, M.P., P. Carriba, M.A. Baltrons, B. Wojciak-Stothard, J.R. Peterson, A. García and E. Galea, Secretase-Independent and RhoGTPase/PAK/ERKDependent Regulation of Cytoskeleton Dynamics in Astrocytes by NSAIDs and Derivatives (4) 1135-1155

Lin, T.-Y., see Kapoor, A. (2) 423-442

Lin, Y., see Murphy, M.P. (1) 135-150

Ling, T.-J., see Yang, S.-G. (1) 107-117

Liu, D., M. Pitta, J.-H. Lee, B. Ray, D.K. Lahiri, K. Furukawa, M. Mughal, H. Jiang, J. Villarreal, R.G. Cutler, N.H. Greig and M.P. Mattson, The $\mathrm{K}_{\mathrm{ATP}}$ Channel Activator Diazoxide Ameliorates Amyloid- $\beta$ and Tau Pathologies and Improves Memory in the 3xTgAD Mouse Model of Alzheimer's Disease (2) 443-457 
Liu, J., L. Chang, F. Roselli, O.F.X. Almeida, X. Gao, X. Wang, D.T. Yew and Y. Wu, Amyloid$\beta$ Induces Caspase-Dependent Loss of PSD-95 and Synaptophysin Through NMDA Receptors (2) 541-556

Liu, R.-T., see Yang, S.-G. (1) 107-117

Liu, Y.-Y. and J.-S. Bian, Hydrogen Sulfide Protects Amyloid- $\beta$ Induced Cell Toxicity in Microglia (4) $1189-1200$

Lizio, R., see Babiloni, C. (4) 1047-1064

Lladó, A., see Fortea, J. (3) 909-922

Lleó, A., see Samaranch, L. (4) 1065-1071

Lopez-Arrieta, J., see Lamberta, J.-C. (1) 247-255

López-Pousa, S., see Garre-Olmo, J. (4) 1157-1167

López-Pousa, S., see Garre-Olmo, J. (4) 1169-1180

Lorenzo, E., see Samaranch, L. (4) 1065-1071

Lotay, N., see Tzimopoulou, S. (4) 1241-1256

Louis, E.D., see Bermejo-Pareja, F. (3) 949-958

Lovblad, K.O., see Haller, S. (1) 315-327

Love, S., see Tayler, H. (4) 1363-1373

Lovestone, S., see Paajanen, T. (4) 1089-1097

Lovestone, S., see Thambisetty, M. (4) 1099-1109

Lovestone, S., see Westman, E. (1) 171-181

Loy, C., see Schofield, E.C. (3) 981-984

Lu, J., see Tokita, Y. (4) 1351-1361

Lu, Q., K. Ding, M.P. Frosch, S. Jones, M. Wolfe, W. Xia and G.W. Lanford, Alzheimer's DiseaseLinked Presenilin Mutation (PS1M146L) Induces Filamin Expression and $\gamma$-Secretase Independent Redistribution (1) 235-245

Luchsinger, J.A., see Gu, Y. (2) 483-492

Luque, R.M., see Gahete, M.D. (3) 819-828

Lynham, S., see Thambisetty, M. (4) 1099-1109

Maestú, F., see Bajo, R. (1) 183-193

Maetzler, W., V. Stoycheva, B. Schmid, C. Schulte, A.K. Hauser, K. Brockmann, A. Melms, T. Gasser and D. Berg, Neprilysin Activity in Cerebrospinal Fluid is Associated with Dementia and Amyloid$\beta_{42}$ Levels in Lewy Body Disease (3) 933-938

Maheshwari, M., see Ellis, G. (2) 593-607

Maier, W., see Popp, J. (2) 459-468

Malek-Ahmadi, M., see Sabbagh, M.N. (3) 1015-1021

Malinovskaya, N.A., see Salmina, A.B. (1) 17-36

Malinverno, M., see Borroni, B. (1) 195-203

Mancini, J., see Didic, M. (4) 1269-1279

Mancuso, M., see Lamberta, J.-C. (1) 247-255

Mandal, P.K. and M. Ahuja, Comprehensive Nuclear Magnetic Resonance Studies on Interactions of Amyloid- $\beta$ with Different Molecular Sized Anesthetics (S3) S27-S34
Mandal, P.K. and V. Fodale, Editorial (S3) S135-S136

Mandal£<P.K.£<see Fodale, V. (S3) S1-S3

Mandal, P.K., see Schifilliti, D. (S3) S35-S41

Mann, D., see Lamberta, J.-C. (1) 247-255

Manson, J., see Tierney, M.C. (4) 1331-1338

Marambaud, P., see Lamberta, J.-C. (1) 247-255

Marcos, A., see Samaranch, L. (4) 1065-1071

Markesbery, W.R., see Ebenezer, P.J. (3) 839-848

Martinez, D., see Ellis, G. (2) 593-607

Martínez-García, A., I. Sastre, M. Recuero, J. Aldudo, E. Vilella, I. Mateo, P. Sánchez-Juan, T. Vargas, E. Carro, F. Bermejo-Pareja, E. RodríguezRodríguez, O. Combarros, M. Rosich-Estrago, A. Frank, F. Valdivieso and M.J. Bullido, PLA2G3, a Gene Involved in Oxidative Stress Induced Death, is Associated with Alzheimer's Disease (4) 11811187

Martínez-Lage, P., see Samaranch, L. (4) 1065-1071

Martins, R.N., see Frost, S. (1) 1-16

Masellis, M., see Gabryelewicz, T. (4) 1123-1133

Masliah, E., see Belinson, H. (3) 959-970

Mason, S.E., A. Noel-Storr and C.W. Ritchie, The Impact of General and Regional Anesthesia on the Incidence of Post-Operative Cognitive Dysfunction and Post-Operative Delirium: A Systematic Review with Meta-Analysis (S3) S67-S79

Massarwa, M., see Israeli-Korn, S.D. (3) 1005-1013

Masters, C.L., see Bahar-Fuchs, A. (4) 1081-1087

Mateo, I., see Lamberta, J.-C. (1) 247-255

Mateo, I., see Martínez-García, A. (4) 1181-1187

Matsumoto, Y., see Tokita, Y. (4) 1351-1361

Matthews, J.C., see Tzimopoulou, S. (4) 1241-1256

Matthews, P.M., see Tzimopoulou, S. (4) 1241-1256

Mattson, M.P., see Liu, D. (2) 443-457

Mazoyer, B., see Zhu, Y.-C. (2) 663-672

Mazzei, D., see Nobili, F. (3) 993-1003

McDowell, I., see Tierney, M.C. (4) 1231-1240

McTiernan, A., see Baker, L.D. (2) 569-579

Mecocci, P., see Paajanen, T. (4) 1089-1097

Mehta, P., see Ji, L. (2) 609-617

Mehta, P.D., see Baker, L.D. (2) 569-579

Mehta, S., see Ji, L. (2) 609-617

Mehta, Y. and R. Singh, Cognitive Dysfunction after Cardiac Surgery (S3) S115-S120

Meininger, V., see Broustal, O. (3) 765-769

Melms, A., see Maetzler, W. (3) 933-938

Melotti, R.M., see Di Nino, G. (S3) S121-S127

Mena, M.Á., J. Perucho, I. Rubio and J.G. de Yébenes, Studies in Animal Models of the Effects of Anesthetics on Behavior, Biochemistry, and Neuronal Cell Death (S3) S43-S48 
Mendonça, V.A., see Diniz, B.S. (4) 1305-1311

Michaelson, D.M., see Belinson, H. (3) 959-970

Milagre, I., M.J. Nunes, M. Moutinho, I. Rivera, A. Fuso, S. Scarpa, M.J. Gama and E. Rodrigues, Chromatin-Modifying Agents Increase Transcription of CYP46A1, a Key Player in Brain Cholesterol Elimination (4) 1209-1221

Milian, M., see Saur, R. (1) 267-284

Millecamps, S., see Broustal, O. (3) 765-769

Miners, J.S., see Tayler, H. (4) 1363-1373

Minthon, L., see Sundelöf, J. (4) 1223-1230

Mistry, P., see Tzimopoulou, S. (4) 1241-1256

Mitchell, A.J., see Benito-León, J. (1) 159-170

Mitchell, A.J., see Cacho, J. (3) 889-896

Mitchell, S.A., see Dangour, A.D. (1) 205-224

Moineddin, R., see Tierney, M.C. (4) 1231-1240

Moineddin, R., see Tierney, M.C. (4) 1331-1338

Molinuevo, J.L., see Fortea, J. (3) 909-922

Molinuevo, J.-L., see Sala-Llonch, R. (2) 523-539

Monfort, P. and V. Felipo, Amyloid- $\beta$ Impairs, and Ibuprofen Restores, the cGMP Pathway, Synaptic Expression of AMPA Receptors and Long-Term Potentiation in the Hippocampus (3) 795-809

Moon, M., see Hong, H.-S. (1) 97-105

Morales, J., see Murphy, M.P. (1) 135-150

Moratti, S., see Bajo, R. (1) 183-193

Morbelli, S., see Nobili, F. (3) 993-1003

Morgan, D.H., see Chen, X. (4) 1289-1303

Morra, A., see Tierney, M.C. (4) 1331-1338

Moss, S., see Bahar-Fuchs, A. (4) 1081-1087

Motawaj, M., K. Peoc'h, J. Callebert and J.-M. Arrang, CSF Levels of the Histamine Metabolite tele-Methylhistamine are only Slightly Decreased in Alzheimer's Disease (3) 861-871

Moutinho, M., see Milagre, I. (4) 1209-1221

Mufson, E.J., see Ginsberg, S.D. (2) 631-639

Mughal, M., see Liu, D. (2) 443-457

Mugueta, C., see Gil-Bea, F.J. (2) 405-413

Mulder, S.D., C.E. Hack, W.M. van der Flier, P. Scheltens, M.A. Blankenstein and R. Veerhuis, Evaluation of Intrathecal Serum Amyloid P (SAP) and CReactive Protein (CRP) Synthesis in Alzheimer's Disease with the Use of Index Values (4) 10731079

Muñoz, F.J., see Ill-Raga, G. (2) 641-652

Muñoz-Fernández, M.Á., see Blanco, A. (2) 493-505

Murphy, D., see Westman, E. (1) 171-181

Murphy, M.P., J. Morales, T.L. Beckett, G. Astarita, D. Piomelli, A. Weidner, C.M. Studzinski, A.L.S. Dowling, X. Wang, H. LeVine, III, R.J. Kryscio, Y. Lin, E. Barrett and E. Head, Changes in Cognition and Amyloid- $\beta$ Processing with Long Term Cholesterol Reduction using Atorvastatin in Aged Dogs (1) 135-150
Murphy, M.P., see Ebenezer, P.J. (3) 839-848

Murray, I.V.J., see Ellis, G. (2) 593-607

Mustafa, M., see Förster, S. (2) 581-591

Mutter, J., A. Curth, J. Naumann, R. Deth and H. Walach, Does Inorganic Mercury Play a Role in Alzheimer's Disease? A Systematic Review and an Integrated Molecular Mechanism (2) 357-374

Na, H.-R., S.-Y. Kim, Y.-H. Chang, M.-H. Park, S.T. Cho, I.-W. Han, T.-Y. Kim and S.-A. Hwang, Functional Assessment Staging (FAST) in Korean Patients with Alzheimer's Disease (1) 151-158

Nacmias, B., see Lamberta, J.-C. (1) 247-255

Naumann, J., see Mutter, J. (2) 357-374

Nevado, A., see Bajo, R. (1) 183-193

Nguyen, D., see Haller, S. (1) 315-327

Nichols, T.E., see Tzimopoulou, S. (4) 1241-1256

Nicolia, V., see Borro, M. (4) 1257-1268

Niwa, M., see Deli, M.A. (3) 777-794

Nixon, R.A., see Ginsberg, S.D. (2) 631-639

Nobili, F., D. Mazzei, B. Dessi, S. Morbelli, A. Brugnolo, P. Barbieri, N. Girtler, G. Sambuceti, G. Rodriguez and M. Pagani, Unawareness of Memory Deficit in Amnestic MCI: FDG-PET Findings (3) 993-1003

Noel-Storr, A., see Mason, S.E. (S3) S67-S69

Nunes, A.F., see Viana, R.J.S. (2) 557-568

Nunes, M.J., see Milagre, I. (4) 1209-1221

Oechsner, M., see Rosengarten, B. (2) 415-421

Offner, H., see Subramanian, S. (2) 619-629

Ogasa, T., see Yang, J. (1) 225-234

Ohta, Y., see Yang, J. (1) 225-234

Ojopi, E.B., see Diniz, B.S. (4) 1305-1311

Okura, Y., see Tokita, Y. (4) 1351-1361

Ono, K., see Wang, J. (2) 653-661

Otto, M., see Steinacker, P. (1) 119-128

Paajanen, T., T. Hänninen, C. Tunnard, P. Mecocci, T. Sobow, M. Tsolaki, B. Vellas, S. Lovestone and H. Soininen for the AddNeuroMed Consortium, CERAD Neuropsychological Battery Total Score in Multinational Mild Cognitive Impairment and Control Populations: The AddNeuroMed Study (4) 1089-1097

Pabst, A., see Steinacker, P. (1) 119-128

Padovani, A., M. Cosseddu, E. Premi, S. Archetti, A. Papetti, C. Agosti, B. Bigni, C. Cerini, B. Paghera, G. Bellelli and B. Borroni, The Speech and Language FOXP2 Gene Modulates the Phenotype of Frontotemporal Lobar Degeneration (3) 923-931 
Padovani, A., see Borroni, B. (1) 195-203 Pagani, M., see Nobili, F. (3) 993-1003

Paghera, B., see Padovani, A. (3) 923-931

Palomer, E., see Ill-Raga, G. (2) 641-652

Panza, F., see Frisardi, V. (3) 715-740

Panza, F., see Lamberta, J.-C. (1) 247-255

Papetti, A., see Padovani, A. (3) 923-931

Parihar, M.S. and G.J. Brewer, Amyloid- $\beta$ as a Modulator of Synaptic Plasticity (3) 741-763

Park, M.-H., see Na, H.-R. (1) 151-158

Parnetti, L., see Borroni, B. (1) 195-203

Parnetti, L., see Chiasserini, D. (4) 1281-1288

Pasinetti, G.M., see Wang, J. (2) 653-661

Pasquier, F., see Lamberta, J.-C. (1) 247-255

Pastor, P., see Samaranch, L. (4) 1065-1071

Patrylo, P.R., see Struble, R.G. (2) 393-399

Pearson, C., see Sabbagh, M.N. (3) 1015-1021

Pedersen, N.L., see Bennet, A.M. (1) 129-134

Penke, B., see Deli, M.A. (3) 777-794

Peoc'h, K., see Motawaj, M. (3) 861-871

Pereda, E., see Bajo, R. (1) 183-193

Pericak-Vance, M.A., see Lamberta, J.-C. (1) 247-255

Perola, M., see Virta, J.J. (3) 939-948

Perry, G., see Borghi, R. (3) 771-775

Perucho, J., see Mena, M.Á. (S3) S43-S48

Peterson, J.R., see Lichtenstein, M.P. (4) 1135-1155

Petrova, M.M., see Salmina, A.B. (1) 17-36

Piccardi, P., see Lamberta, J.-C. (1) 247-255

Piccini, A., see Borghi, R. (3) 771-775

Pievani, M., see Babiloni, C. (4) 1047-1064

Pike, K., see Bahar-Fuchs, A. (4) 1081-1087

Pilotto, A., see Frisardi, V. (3) 715-740

Pilotto, A., see Lamberta, J.-C. (1) 247-255

Piomelli, D., see Murphy, M.P. (1) 135-150

Pitta, M., see Liu, D. (2) 443-457

Plano, A., see Jyoti, A. (3) 873-887

Platt, B., see Jyoti, A. (3) 873-887

Plymate, S.R., see Baker, L.D. (2) 569-579

Pöhler, M., see Rosengarten, B. (2) 415-421

Poncet, M., see Didic, M. (4) 1269-1279

Popp, J., P. Lewczuk, I. Frommann, H. Kölsch, J. Kornhuber, W. Maier and F. Jessen, Cerebrospinal Fluid Markers for Alzheimer's Disease over the Lifespan: Effects of Age and the APOE $\varepsilon 4$ Genotype (2) 459-468

Poppe, M., see Westman, E. (1) 171-181

Porcellini, E., see Lamberta, J.-C. (1) 247-255

Premi, E., see Borroni, B. (1) 195-203

Premi, E., see Padovani, A. (3) 923-931

Prestia, A., V. Drago, P.E. Rasser, M. Bonetti, P.M. Thompson and G.B. Frisoni, Cortical Changes in Incipient Alzheimer's Disease (4) 1339-1349
Prince, J.A., see Bennet, A.M. (1) 129-134

Quinn, J.F., see Subramanian, S. (2) 619-629

Quinn, J.G., see Coulson, D.T.R. (4) 1111-1122

Rabiner, E.A., see Tzimopoulou, S. (4) 1241-1256

Rabinovich-Toidman, P., see Arbel-Ornath, M. (2) 469-482

Rafferty, K., see Dangour, A.D. (1) 205-224

Räihä, I., see Virta, J.J. (3) 939-948

Ramalho, R.M., see Viana, R.J.S. (2) 557-568

Rami, L., see Sala-Llonch, R. (2) 523-539

Ramirez, M.J., see Gil-Bea, F.J. (2) 405-413

Ramírez, M.J., see Gil-Bea, F.J. (3) 829-838

Rammes, G., see Eckel, B. (S3) S21-S26

Ramos-Fernández, E., see Ill-Raga, G. (2) 641-652

Ranjeva, J.-P., see Didic, M. (4) 1269-1279

Rasmussen, L.S.£< see Fodale, V. (S3) S1-S3

Rasmussen, L.S., see Funder, K.S. (S3) S129-S134

Rasser, P.E., see Prestia, A. (4) 1339-1349

Ravaglia, G., see Lamberta, J.-C. (1) 247-255

Ravid, R., see Cao, Y. (1) 329-342

Ravid, R., see Coulson, D.T.R. (4) 1111-1122

Ray, B., see Liu, D. (2) 443-457

Recuero, M., see Martínez-García, A. (4) 1181-1187

Reesink, F.E., A.W. Lemstra, K.D. van Dijk, H.W. Berendse, W.D.J. van de Berg, M. Klein, M.A. Blankenstein, P. Scheltens, M.M. Verbeek and W.M. van der Flier, CSF $\alpha$-Synuclein Does Not Discriminate Dementia with Lewy Bodies from Alzheimer's Disease (1) 87-95

Reiman, E.M., see Tzimopoulou, S. (4) 1241-1256

Reiman, E.M., see Valla, J. (1) 307-313

Reiser, M.F., see Teipel, S.J. (2) 507-522

Resnick, S.M., see Thambisetty, M. (4) 1099-1109

Reuter, I., see Rosengarten, B. (2) 415-421

Reynolds, C.A., see Bennet, A.M. (1) 129-134

Richard, E., W.A. van Gool, J.J.M. Hoozemans, E.S. van Haastert, P. Eikelenboom, A.J.M. Rozemuller and W.D.J. van de Berg, Morphometric Changes in the Cortical Microvascular Network in Alzheimer's Disease (3) 811-818

Richard, F., see Lamberta, J.-C. (1) 247-255

Riddoch-Contreras, J., see Thambisetty, M. (4) 10991109

Riedel, G., see Jyoti, A. (3) 873-887

Rinne, J.O., see Virta, J.J. (3) 939-948

Ritchie, C.W., see Mason, S.E. (S3) S67-S69

Ritchie, K., see Ancelin, M.-L. (S3) S105-S113

Ritchie, K., see Fodale, V. (S3) S1-S3

Rivera, I., see Milagre, I. (4) 1209-1221 
Rodrigues, C.M.P., see Viana, R.J.S. (2) 557-568

Rodrigues, E., see Milagre, I. (4) 1209-1221

Rodriguez, C., see Haller, S. (1) 315-327

Rodriguez, G., see Nobili, F. (3) 993-1003

Rodríguez-Rodríguez, E., see Martínez-García, A. (4) 1181-1187

Rogaeva, E., see Gabryelewicz, T. (4) 1123-1133

Roher, A.E., see Valla, J. (1) 307-313

Roltsch, E., see Ellis, G. (2) 593-607

Romano, D., see Zhang, C. (2) 683-694

Rominger, A., see Förster, S. (2) 581-591

Rosa, G., see Bilotta, F. (S3) S81-S89

Rosa, G., see Di Nino, G. (S3) S121-S127

Rosa, G., see Schifilliti, D. (S3) S35-S41

Roselli, F., see Liu, J. (2) 541-556

Rosengarten, B., V. Dannhardt, O. Burr, M. Pöhler, S. Rosengarten, M. Oechsner and I. Reuter, Neurovascular Coupling in Parkinson's Disease Patients: Effects of Dementia and Acetylcholinesterase Inhibitor Treatment (2) 415-421

Rosengarten, S., see Rosengarten, B. (2) 415-421

Rosich-Estrago, M., see Martínez-García, A. (4) 11811187

Ross, J.S. and B.P. Imbimbo, Are $\gamma$-secretase inhibitors detrimental for Alzheimer's disease patients? (2) 401-404

Rossini, P.M., see Babiloni, C. (4) 1047-1064

Rowe, C., see Bahar-Fuchs, A. (4) 1081-1087

Rozemuller, A.J.M., see Richard, E. (3) 811-818

Rubio, A., see Gahete, M.D. (3) 819-828

Rubio, I., see Mena, M.Á. (S3) S43-S48

Ruiz, A., see Lamberta, J.-C. (1) 247-255

Run, X., Z. Liang and C.-X. Gong, Anesthetics and Tau Protein: Animal Model Studies (S3) S49-S55

Sabbagh, M.N., M. Malek-Ahmadi, R. Kataria, C.M. Belden, D.J. Connor, C. Pearson, S. Jacobson, K. Davis, R. Yaari and U. Singh, The Alzheimer's Questionnaire: A Proof of Concept Study for a New Informant-Based Dementia Assessment (3) 1015-1021

Saint-Pol, J., see Candela, P. (3) 849-859

Salachas, F., see Broustal, O. (3) 765-769

Sala-Llonch, R., B. Bosch, E.M. Arenaza-Urquijo, L. Rami, N. Bargalló, C. Junqué, J.-L. Molinuevo and D. Bartrés-Faz, Greater Default-Mode Network Abnormalities Compared to High Order Visual Processing Systems in Amnestic Mild Cognitive Impairment: An Integrated Multi-Modal MRI Study (2) 523-539

Sala-Llonch, R., see Fortea, J. (3) 909-922
Salmina, A.B., A.I. Inzhutova, N.A. Malinovskaya and M.M. Petrova, Endothelial Dysfunction and Repair in Alzheimer-Type Neurodegeneration: Neuronal and Glial Control (1) 17-36

Salmon, E., see Collette, F. (3) 897-907

Samaranch, L., S. Cervantes, A. Barabash, A. Alonso, J.A. Cabranes, I. Lamet, I. Ancín, E. Lorenzo, P. Martínez-Lage, A. Marcos, J. Clarimón, D. Alcolea, A. Lleó, R. Blesa, T. Gómez-Isla and P. Pastor, The Effect of MAPT $\mathrm{H} 1$ and $A P O E \varepsilon 4$ on Transition from Mild Cognitive Impairment to Dementia (4) 1065-1071

Sambuceti, G., see Nobili, F. (3) 993-1003

Sánchez-Juan, P., see Martínez-García, A. (4) 11811187

Sánchez-Valle, R., see Fortea, J. (3) 909-922

Sancho, M., see Bajo, R. (1) 183-193

Santa-Maria, I., see Wang, J. (2) 653-661

Santamaria, L.B., see Schifilliti, D. (S3) S35-S41

Sarazin, M., V. Chauviré, E. Gerardin, O. Colliot, S. Kinkingnéhun, L.C. de Souza, L. HugonotDiener, L. Garnero, S. Lehéricy, M. Chupin and B. Dubois, The Amnestic Syndrome of Hippocampal in Alzheimer's Disease: An MRI Study (1) 285-294

Sastre, I., see Martínez-García, A. (4) 1181-1187

Saur, R., M. Milian, M. Erb, G.W. Eschweiler, W. Grodd and T. Leyhe, Cortical Activation during Clock Reading as a Quadratic Function of Dementia State (1) 267-284

Savage, G., see Bahar-Fuchs, A. (4) 1081-1087

Scali, J., see Ancelin, M.-L. (S3) S105-S113

Scarmeas, N., see Gu, Y. (2) 483-492

Scarpa, S., see Borro, M. (4) 1257-1268

Scarpa, S., see Milagre, I. (4) 1209-1221

Scarpini, E., see Lamberta, J.-C. (1) 247-255

Schechtman, E., see Israeli-Korn, S.D. (3) 1005-1013

Scheltens, P., see Mulder, S.D. (4) 1073-1079

Scheltens, P., see Reesink, F.E. (1) 87-95

Schifilliti, D., L.B. Santamaria, G. Rosa, G. Di Nino, P.K. Mandal and V. Fodale, Cholinergic Central System, Alzheimer's Disease, and Anesthetics Liaison: A Vicious Circle? (S3) S35-S41

Schjeide, B.-M.M., see Lamberta, J.-C. (1) 247-255

Schmid, B., see Maetzler, W. (3) 933-938

Schofield, E.C., G.M. Halliday, J. Kwok, C. Loy, K.L. Double and J.R. Hodges, Low Serum Progranulin Predicts the Presence of Mutations: A Prospective Study (3) 981-984

Schulte, C., see Maetzler, W. (3) 933-938

Searle, G., see Tzimopoulou, S. (4) 1241-1256 
Seripa, D., see Frisardi, V. (3) 715-740

Seripa, D., see Lamberta, J.-C. (1) 247-255

Sevin, E., see Candela, P. (3) 849-859

Siciliano, G., see Lamberta, J.-C. (1) 247-255

Simmaco, M., see Borro, M. (4) 1257-1268

Simmons, A., see Westman, E. (1) 171-181

Simpkins, J.W., see Ill-Raga, G. (2) 641-652

Singh, R., see Mehta, Y. (S3) S115-S120

Singh, U., see Sabbagh, M.N. (3) 1015-1021

Sipos, Á., see Deli, M.A. (3) 777-794

Sleegers, K., see Lamberta, J.-C. (1) 247-255

Small, D.H., see Vincent, A.J. (3) 699-714

Smith, L., see Dangour, A.D. (1) 205-224

Smith, M.A., see Borghi, R. (3) 771-775

Sobow, T., see Paajanen, T. (4) 1089-1097

Soininen for the AddNeuroMed Consortium, H., see Paajanen, T. (4) 1089-1097

Soininen, H., see Lamberta, J.-C. (1) 247-255

Sojkova, J., see Thambisetty, M. (4) 1099-1109

Solas, M., see Gil-Bea, F.J. (2) 405-413

Solas, M., see Gil-Bea, F.J. (3) 829-838

Solfrizzi, V., see Frisardi, V. (3) 715-740

Solfrizzi, V., see Lamberta, J.-C. (1) 247-255

Solomon, A., see Gil-Bea, F.J. (2) 405-413

Solomon, A., see Gil-Bea, F.J. (3) 829-838

Solomon, B., see Arbel-Ornath, M. (2) 469-482

Son, S.-M., see Hong, H.-S. (1) 97-105

Sorbi, S., see Lamberta, J.-C. (1) 247-255

Soumaré, A., see Zhu, Y.-C. (2) 663-672

Spalletta, G., see Lamberta, J.-C. (1) 247-255

Spenger, C., see Westman, E. (1) 171-181

Stazi, E., see Bilotta, F. (S3) S81-S89

Steer, C.J., see Viana, R.J.S. (2) 557-568

Steinacker, P., H. Klafki, S. Lehnert, S. Jesse, C.A.F.v. Arnim, H. Tumani, A. Pabst, H.A. Kretzschmar, J. Wiltfang and M. Otto, ERK2 is Increased in Cerebrospinal Fluid of Creutzfeldt-Jakob Disease Patients (1) 119-128

Steinbach, S., see Förster, S. (2) 581-591

Steinmetz, J., see Funder, K.S. (S3) S129-S134

Stern, Y., see Gu, Y. (2) 483-492

Stevenson, J.A., see Zhang, C. (2) 683-694

Stoycheva, V., see Maetzler, W. (3) 933-938

Struble, R.G., T. Ala, P.R. Patrylo, G.J. Brewer and X.X. Yan, Is Brain Amyloid Production a Cause or a Result of Dementia of The Alzheimer's Type? (2) 393-399

Strugatsky, R., see Israeli-Korn, S.D. (3) 1005-1013

Studzinski, C.M., see Murphy, M.P. (1) 135-150

Subramanian, S., P. Ayala, T.L. Wadsworth, C.J. Harris, A.A. Vandenbark, J.F. Quinn and H. Offner, CCR6: A Biomarker for Alzheimer's-like Disease in a Triple Transgenic Mouse Model (2) 619-629
Sun, X.-S., see Yang, S.-G. (1) 107-117

Sundelöf, J., J. Sundström, O. Hansson, M. EriksdotterJönhagen, V. Giedraitis, A. Larsson, M. DegermanGunnarsson, M. Ingelsson, L. Minthon, K. Blennow, L. Kilander, H. Basun and L. Lannfelt, Higher Cathepsin B Levels in Plasma in Alzheimer's Disease Compared to Healthy Controls (4) 1223-1230

Sundström, J., see Sundelöf, J. (4) 1223-1230

Szalai, A., see Deli, M.A. (3) 777-794

Tabaton, M., see Borghi, R. (3) 771-775

Tajes, M., see Ill-Raga, G. (2) 641-652

Talib, L.L., see Diniz, B.S. (4) 1305-1311

Tamagno, E., see Borghi, R. (3) 771-775

Tanzi, R.E., see Lamberta, J.-C. (1) 247-255

Tanzi, R.E., see Zhang, C. (2) 683-694

Tayler, H., T. Fraser, J.S. Miners, P.G. Kehoe and S. Love, Oxidative Balance in Alzheimer's Disease: Relationship to APOE, Braak Tangle Stage, and the Concentrations of Soluble and Insoluble Amyloid- $\beta$ (4) 1363-1373

Teipel, S.J., see Förster, S. (2) 581-591

Teipel, S.J., T. Meindl, M. Wagner, B. Stieltjes, S. Reuter, K.-H. Hauenstein, M. Filippi, U. Ernemann, M.F. Reiser and H. Hampel, Longitudinal Changes in Fiber Tract Integrity in Healthy Aging and Mild Cognitive Impairment: A DTI FollowUp Study (2) 507-522

Teixeira, A.L., see Diniz, B.S. (4) 1305-1311

Teplow, D.B., see Wang, J. (2) 653-661

Teramukai, S., see Zhou, B. (2) 673-682

Thambisetty, M., R. Tripaldi, J. Riddoch-Contreras, A. Hye, Y. An, J. Campbell, J. Sojkova, A. Kinsey, S. Lynham, Y. Zhou, L. Ferrucci, D.F. Wong, S. Lovestone and S.M. Resnick, Proteome-Based Plasma Markers of Brain Amyloid- $\beta$ Deposition in Non-Demented Older Individuals (4) 1099_ 1109

Thompson, P.M., see Prestia, A. (4) 1339-1349

Tierney, M.C., R. Moineddin and I. McDowell, Prediction of All-Cause Dementia Using Neuropsychological Tests within 10 and 5 Years of Diagnosis in a Community-Based Sample (4) 1231-1240

Tierney, M.C., R. Moineddin, A. Morra, J. Manson and J. Blake, Intensity of Recreational Physical Activity throughout Life and Later Life Cognitive Functioning in Women (4) 1331-1338

Timmer, N.M., H.B. Kuiperij, R.M.W. de Waal and M.M. Verbeek, Do Amyloid- $\beta$-associated Factors Co-deposit with $\mathrm{A} \beta$ in Mouse Models for Alzheimer's Disease? (2) 345-355 
Titi, L., see Bilotta, F. (S3) S81-S89

Tognoni, G., see Lamberta, J.-C. (1) 247-255

Tokita, Y., K. Kaji, J. Lu, Y. Okura, K. Kohyama and Y. Matsumoto, Assessment of Non-Viral Amyloid$\beta$ DNA Vaccines on Amyloid- $\beta$ Reduction and Safety in Rhesus Monkeys (4) 1351-1361

Tóth, A., see Deli, M.A. (3) 777-794

Townsend, K., see Chen, S. (4) 1313-1329

Trincado, R., see Bermejo-Pareja, F. (3) 949-958

Tripaldi, R., see Thambisetty, M. (4) 1099-1109

Tripathi, M. and D. Vibha, Unusual Risk Factors for Cognitive Decline (S3) S57-S65

Tsolaki, M., see Paajanen, T. (4) 1089-1097

Tumani, H., see Steinacker, P. (1) 119-128

Tunnard, C., see Paajanen, T. (4) 1089-1097

Turner, A.J., see Whitehouse, I.J. (3) 1023-1031

Tzimopoulou, S., V.J. Cunningham, T.E. Nichols, G. Searle, N.P. Bird, P. Mistry, I.J. Dixon, W.A. Hallett, B. Whitcher, A.P. Brown, M. Zvartau-Hind, N. Lotay, R.Y.K. Lai, M. Castiglia, B. Jeter, J.C. Matthews, K. Chen, D. Bandy, E.M. Reiman, M. Gold, E.A. Rabiner and P.M. Matthews, A MultiCenter Randomized Proof-of-Concept Clinical Trial Applying [ $\left.{ }^{18} \mathrm{~F}\right]$ FDG-PET for Evaluation of Metabolic Therapy with Rosiglitazone XR in Mild to Moderate Alzheimer's Disease (4) 1241-1256

Tzourio, C., see Lamberta, J.-C. (1) 247-255

Tzourio, C., see Zhu, Y.-C. (2) 663-672

Vaitl, A., see Förster, S. (2) 581-591

Valdivieso, F., see Lamberta, J.-C. (1) 247-255

Valdivieso, F., see Martínez-García, A. (4) 1181-1187

Valla, J., R. Yaari, A.B. Wolf, Y. Kusne, T.G. Beach, A.E. Roher, J.J. Corneveaux, M.J. Huentelman, R.J. Caselli and E.M. Reiman, Reduced Posterior Cingulate Mitochondrial Activity in Expired Young Adult Carriers of the APOE $\varepsilon 4$ Allele, the Major Late-Onset Alzheimer's Susceptibility Gene (1) 307-313

Van Broeckhoven, C., see Lamberta, J.-C. (1) 247-255 van de Berg, W.D.J., see Reesink, F.E. (1) 87-95 van de Berg, W.D.J., see Richard, E. (3) 811-818 van der Flier, W.M., see Mulder, S.D. (4) 1073-1079 van der Flier, W.M., see Reesink, F.E. (1) 87-95 Van der Linden, M., see Collette, F. (3) 897-907 van Dijk, K.D., see Reesink, F.E. (1) 87-95 van Gool, W.A., see Richard, E. (3) 811-818 van Haastert, E.S., see Richard, E. (3) 811-818 Vandenbark, A.A., see Subramanian, S. (2) 619-629 Vanderweyde, T., M.M. Bednar, S.A. Forman and B. Wolozin, Iatrogenic Risk Factors for Alzheimer's Disease: Surgery and Anesthesia (S3) S91-S104
Vargas, T., see Martínez-García, A. (4) 1181-1187

Vecchio, F., see Babiloni, C. (4) 1047-1064

Veerhuis, R., see Mulder, S.D. (4) 1073-1079

Vega, S., see Benito-León, J. (1) 159-170

Vellas, B., see Dangour, A.D. (1) 205-224

Vellas, B., see Paajanen, T. (4) 1089-1097

Vendemiale, G., see Frisardi, V. (3) 715-740

Vepsäläinen, S., see Lamberta, J.-C. (1) 247-255

Verbeek, M.M., see Reesink, F.E. (1) 87-95

Verbeek, M.M., see Timmer, N.M. (2) 345-355

Veszelka, S., see Deli, M.A. (3) 777-794

Viana, R.J.S., R.M. Ramalho, A.F. Nunes, C.J. Steer and C.M.P. Rodrigues, Modulation of Amyloid$\beta$ Peptide-Induced Toxicity through Inhibition of JNK Nuclear Localization and Caspase-2 Activation (2) 557-568

Vibha, D., see Tripathi, M. (S3) S57-S65

Vicente-Villardón, J.L., see Cacho, J. (3) 889-896

Vilalta-Franch, J., see Garre-Olmo, J. (4) 1157-1167

Vilalta-Franch, J., see Garre-Olmo, J. (4) 1169-1180

Vilarrasa, A.B., see Garre-Olmo, J. (4) 1157-1167

Vilarrasa, A.B., see Garre-Olmo, J. (4) 1169-1180

Vilella, E., see Martínez-García, A. (4) 1181-1187

Villarejo, A., see Bermejo-Pareja, F. (3) 949-958

Villarreal, J., see Liu, D. (2) 443-457

Villemagne, V.L., see Bahar-Fuchs, A. (4) 1081-1087

Vincent, A.J., R. Gasperini, L. Foa and D.H. Small, Astrocytes in Alzheimer's Disease: Emerging Roles in Calcium Dysregulation and Synaptic Plasticity (3) 699-714

Virta, J.J., T. Järvenpää, K. Heikkilä, M. Perola, M. Koskenvuo, I. Räihä, J.O. Rinne and J. Kaprio, Midlife Alcohol Consumption and Later Risk of Cognitive Impairment: A Twin Follow-up Study (3) 939-948

Wadsworth, T.L., see Subramanian, S. (2) 619-629

Wagener, J.F., see Chen, X. (4) 1289-1303

Wahlund, L.-O., see Westman, E. (1) 171-181

Walach, H., see Mutter, J. (2) 357-374

Wang, B.-J., see Kapoor, A. (2) 423-442

Wang, J., I. Santa-Maria, L. Ho, H. Ksiezak-Reding, K. Ono, D.B. Teplow and G.M. Pasinetti, Grape Derived Polyphenols Attenuate Tau Neuropathology in a Mouse Model of Alzheimer's Disease (2) 653-661

Wang, L., see Yang, S.-G. (1) 107-117

Wang, X., see Liu, J. (2) 541-556

Wang, X., see Murphy, M.P. (1) 135-150

Watson, G.S., see Baker, L.D. (2) 569-579

Wegiel, J., see Ji, L. (2) 609-617 
Weidner, A., see Murphy, M.P. (1) 135-150

Weidner, A.M., see Ebenezer, P.J. (3) 839-848

Westman, E., L.-O. Wahlund, C. Foy, M. Poppe, A. Cooper, D. Murphy, C. Spenger, S. Lovestone and A. Simmons, Combining MRI and MRS to Distinguish Between Alzheimer's Disease and Healthy Controls (1) 171-181

Whitcher, B., see Tzimopoulou, S. (4) 1241-1256

Whitehouse, I.J., C. Jackson, A.J. Turner and N.M. Hooper, Prion Protein is Reduced in Aging and in Sporadic but not in Familial Alzheimer's Disease (3) 1023-1031

Whitehouse, P.J., see Dangour, A.D. (1) 205-224

Wilkinson, C.W., see Baker, L.D. (2) 569-579

Wiltfang, J., see Steinacker, P. (1) 119-128

Winblad, B., E. Giacobini, L. Frölich, L.T. Friedhoff, G. Bruinsma, R.E. Becker and N.H. Greig, Phenserine Efficacy in Alzheimer's Disease (4) 1201-1208

Winblad, B., see Gil-Bea, F.J. (2) 405-413

Winblad, B., see Gil-Bea, F.J. (3) 829-838

Wojciak-Stothard, B., see Lichtenstein, M.P. (4) 11351155

Wolf, A.B., see Valla, J. (1) 307-313

Wolfe, M., see Lu, Q. (1) 235-245

Wolozin, B., see Vanderweyde, T. (S3) S91-S104

Wong, D.F., see Thambisetty, M. (4) 1099-1109

Wszolek, Z., see Gabryelewicz, T. (4) 1123-1133

Wu, G.-H., see Kapoor, A. (2) 423-442

Wu, J., see Yang, J. (1) 225-234

Wu, Y., see Liu, J. (2) 541-556

Wuu, J., see Ginsberg, S.D. (2) 631-639

Xia, W., see Lu, Q. (1) 235-245

Xiao, Y., see Cao, Y. (1) 329-342

Xie, Z., see Zhang, C. (2) 683-694

Xue, D., see Yang, S.-G. (1) 107-117

Yaari, R., see Sabbagh, M.N. (3) 1015-1021

Yaari, R., see Valla, J. (1) 307-313

Yakushev, I., see Förster, S. (2) 581-591

Yan, X.-X., see Struble, R.G. (2) 393-399

Yang, J., T. Ogasa, Y. Ohta, K. Abe and J. Wu, Decline of Human Tactile Angle Discrimination in Patients with Mild Cognitive Impairment and Alzheimer's Disease (1) 225-234
Yang, Q., see Yu, C.-J. (1) 257-266

Yang, S.-G., X. Zhang, X.-S. Sun, T.-J. Ling, Y. Feng, X.-Y. Du, M. Zhao, Y. Yang, D. Xue, L. Wang and R.-T. Liu, Diverse Ecdysterones Show Different Effects on Amyloid- $\beta_{42}$ Aggregation but All Uniformly Inhibit Amyloid- $\beta_{42}$-Induced Cytotoxicity (1) 107-117

Yang, Y., see Yang, S.-G. (1) 107-117

Yen, C.-T., see Kapoor, A. (2) 423-442

Yew, D.T., see Liu, J. (2) 541-556

Yoshiyama, Y., A. Kojima, C. Ishikawa and K. Arai, Anti-Inflammatory Action of Donepezil Ameliorates Tau Pathology, Synaptic Loss, and Neurodegeneration in a Tauopathy Mouse Model (1) 295306

Yu, C.-J., M.-F. Zheng, C.-X. Kuang, W.-D. Huang and Q. Yang, Oren-Gedoku-to and Its Constituents with Therapeutic Potential in Alzheimer's Disease Inhibit Indoleamine 2, 3-Dioxygenase Activity In Vitro (1) 257-266

Zekanowski, C., see Gabryelewicz, T. (4) 1123-1133

Zelenika, D., see Lamberta, J.-C. (1) 247-255

Zetterberg, H., see Chiasserini, D. (4) 1281-1288

Zhang, C., A. Browne, J.R. DiVito, J.A. Stevenson, D. Romano, Y. Dong, Z. Xie and R.E. Tanzi, Amyloid- $\beta$ Production Via Cleavage of Amyloid$\beta$ Protein Precursor is Modulated by Cell Density (2) 683-694

Zhang, L., see Ebenezer, P.J. (3) 839-848

Zhang, X., see Yang, S.-G. (1) 107-117

Zhao, M., see Yang, S.-G. (1) 107-117

Zhao, Q., see Zhou, B. (2) 673-682

Zheng, M.-F., see Yu, C.-J. (1) 257-266

Zhou, B., Q. Zhao, S. Teramukai, D. Ding, Q. Guo, M. Fukushima and Z. Hong, Executive Function Predicts Survival in Alzheimer Disease: A Study in Shanghai (2) 673-682

Zhou, Y., see Thambisetty, M. (4) 1099-1109

Zhu, Y.-C., C. Dufouil, A. Soumaré, B. Mazoyer, H. Chabriat and C. Tzourio, High Degree of Dilated Virchow-Robin Spaces on MRI is Associated with Increased Risk of Dementia (2) 663-672

Zimmer, D., see Ellis, G. (2) 593-607

Zvartau-Hind, M., see Tzimopoulou, S. (4) 1241-1256 